\title{
Implementation of a Multisensory Strategy for Emotion Recognition
}

\author{
Hamilton Rivera*, Carlos Valadão*, Eliete Caldeira*, Teodiano Bastos**** \\ *Postgraduate Program in Electrical Engineering, Assistive Technology Group, \\ **Postgraduate Program in Biotechnology, Assistive Technology Group, \\ hamriver@gmail.com,carlostvaladao@gmail.com,eliete.caldeira@ufes.br,teodiano.bastos@ufes.br \\ Federal University of Espirito Santo, Vitoria, Brazil.
}

\begin{abstract}
Multisensorial emotion recognition methods require several sensors to collect relevant data from expressions, as these systems are more complex than a single sensor in terms of number and diversity of sensors involved, and computational complexity of data-interpreting algorithms. This work presents a multisensorial integration strategy for emotions recognition, with three methods of integration implemented, which are Decision-Level, Feature-Level and Hybrid-Level. The advantage of such multisensorial system was the three sensors (eye tracker, Kinect and thermal camera) combined lead to a better and varied analysis of emotional aspects, allowing the evaluation of focal attention, valence and arousal detection, and emotion recognition. This system also presents the potential to analyze people's emotions by facial features using contactless sensors in semi-structured environments, such as clinics, laboratories, or classrooms.
\end{abstract}

Keywords: Multisensorial integration; emotion recognition; social visual attention; valence detection; arousal detection; facial expression recognition.

\section{INTRODUCTION}

One of the challenging issues in affective computing is to endow a machine with an emotional intelligence. Humans employ multiple sensors in emotion recognition. At the same way, an emotionally intelligent system requires multiples sensors to be able to create an affective interaction with users. Many factors render multisensorial emotion recognition approaches appealing. First, humans employ a multisensorial approach in emotion recognition, then, machines attempt to reproduce elements of the human emotional intelligence. Second, the combination of multiple-affective signals not only provides a richer collection of data, but also helps alleviating the effects of uncertainty in raw signals.

A multisensorial approach presents challenges associated with the fusion of single signals, dimensionality of the feature space, and incompatibility of collected signals in terms of time, resolution and format. With these multimodal emotion recognition approaches, information extracted from each modality is reconciled to obtain a single emotion classification result, which is known as multimodal integration. The literature on this topic is rich and generally describes three types of integration mechanisms: featurelevel, decision-level, and hybrid approaches.

A common method to perform modality integration is to create a single set from all collected features, and, thus, a single classifier is trained on the feature set. However, feature-level integration is plagued by several challenges: first, multimodal feature set contains more information than an unimodal one, which can present difficulties if the training dataset is limited. In fact, Hughes (1968) has proven that the increase in the feature set may decrease classification accuracy if the training set is not large enough. Second, features from various modalities are collected at different time scales (Pantic, 2003). A large feature set undoubtedly increases the computational load of the classification algorithm (Lingenfelser, 2011). Finally, one of the advantages of multimodal emotion recognition is the ability of synchronizing data easily and producing an emotion classification result in the presence of missing or corrupted data. However, feature-level integration is more vulnerable to the latter issues than decision-level integration techniques (Wagner, 2011).

Typically, an emotion recognition system produces errors in some area of the feature space (Alexandre, 2001). Hence, combining the results of multiple systems can alleviate this shortcoming. This is especially true when each system is operating on a different modality that corresponds to a separate feature space. Using decision-level integration, modalities can be independently classified using separate models, and the results are joined using a multitude of possible methods. Therefore, this approach is said to employ an ensemble of systems and classifiers. Ensemble members can belong to the same family or different families of statistical classifiers. In fact, static and dynamic classifiers can both be employed in such multimodal system.

When an integration technique combines feature and decision-level integration, it is referred to as a hybrid-integration scheme. For instance, we can achieve integration in two stages. In the first stage, a system can perform feature-level integration. For example, a single classifier can handle features from audio and video signals. In the second stage, decision-level integration can be used to combine the results of that with another one operating on 
physiological features. Kim (2005) proposes a simple hybrid-integration approach where the result from the feature-level integration is fed as an additional input to the decision-level integration stage.

In Rivera (2017), a multisensorial system for emotions recognition was introduced. The system is based on the integration of three sensors (Kinect, eye tracker and thermal camera). The proposed strategy for integration of these sensors was designed to improve the emotion recognition system, which is based on the detection of focus of attention, expression recognition and thermal variation. The technique used for detection of focus of attention was IR-pupil corneal reflection (IR-PCR) introduced by Duchowski (2000) and Bengoechea et al. (2012), which provides highly accurate gaze point measurements, of up to $0.5^{\circ}$ of visual angle. For expression recognition, the implementation was based on Facial Action Coding System (FACS), that describes all possible perceivable facial muscle movements in terms of predefined action units (AUs) proposed by Ekman and Friesen (1978) and implemented in different researches (Mase, 1991; Essa, 1997; and Bartlett et al., 1999). For detection of thermal variation, two techniques were implemented: Facial Thermal - Region of Interest (FT-RoI) introduced by Veltman and Vos (2005) and Khan, Ward, and Ingleby (2009), and Facial Thermal Feature Points (FTFP), which have been tried by Khan et al. (2005), Pavlidis (2004) and Sugimoto (2000). Fig. 1 shows the block diagram of the multisensorial system for emotion recognition.

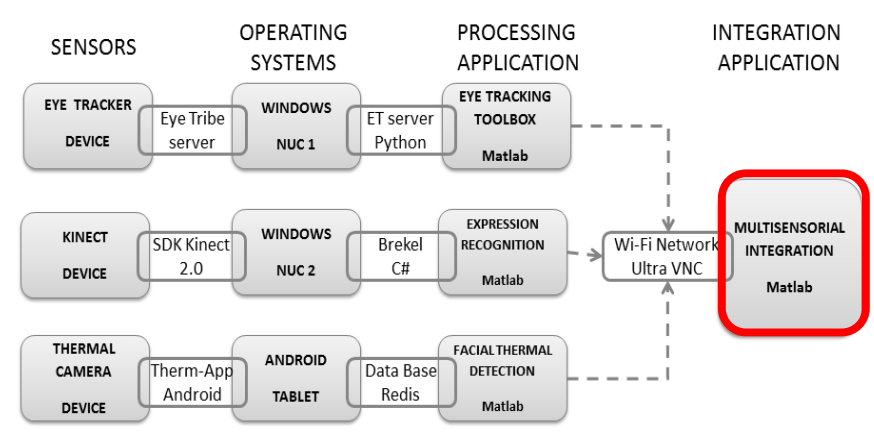

Fig. 1. Block diagram of the multisensorial system for emotion recognition.

\section{PURPOSE OF THIS WORK}

In this paper, the multisensorial integration strategies implemented in Rivera (2017) are presented: in the first stage of the system, eye tracker and Kinect were integrated using a decision-level technique; and a feature-level technique was used to integrate thermal camera and Kinect in the second stage; during the third stage, a hybrid-level technique was used to integrate thermal camera, eye tracker and Kinect. In order to obtain a more complete and varied analysis of the emotional aspects, allowing to evaluating focal attention, valence comprehension, valence expressions, facial expression, valence recognition and arousal recognition. Fig. 2 shows the block diagram of the three integration levels implemented in this research.

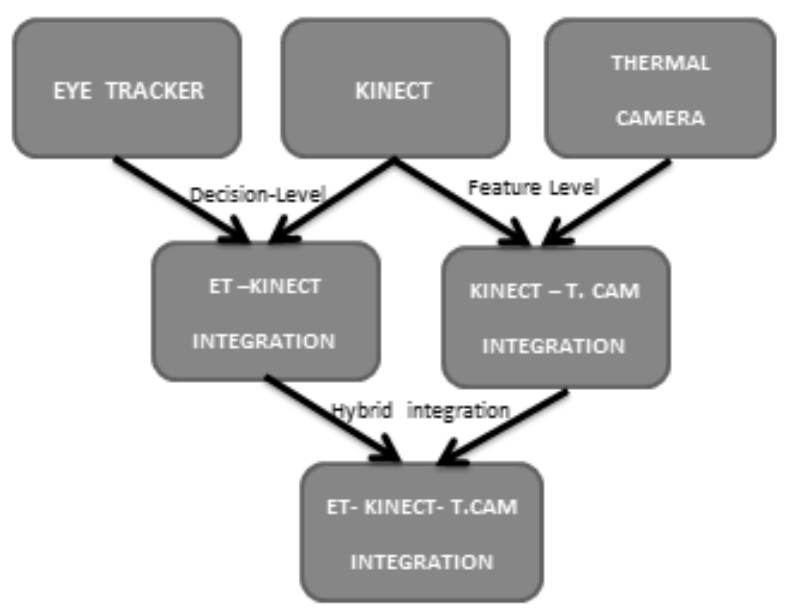

Fig. 2. Block diagram of the proposed multisensorial integration strategy.

\section{MATERIALS}

The experimental platform implemented in this work is composed of one Microsoft Kinect 2.0 device, which provides high quality color and depth images used to obtain facial points (FACS-AU); one Opgal App-therm thermal camera, used to determine the Region of Interest (RoI) in the thermal image, so that the features of the face can be extracted; one eye tracker device for gaze tracking; two minicomputer for image and data processing; one tablet for thermal image processing, and a desktop computer, for offline processing and reporting of results.
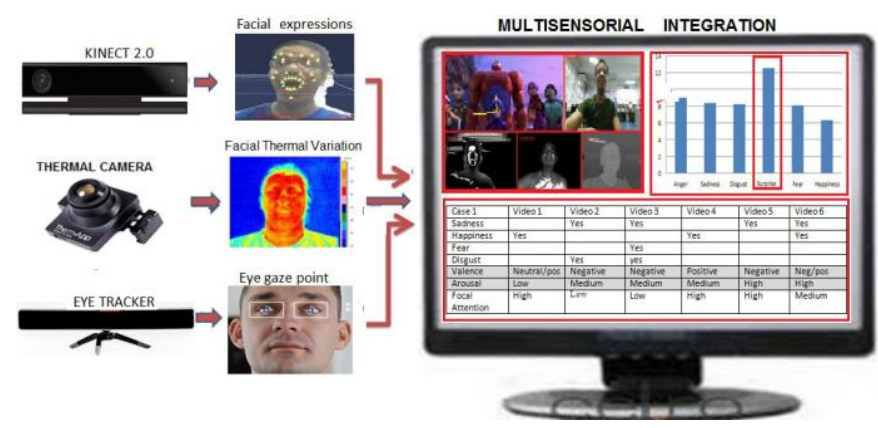

Fig. 3. Multisensorial integration: thermal camera, Kinect and eye tracker.

\section{ENVIRONMENT FOR EXPERIMENTAL TEST}

To set up the experimental tests, the platform shown in Fig. 4 was used. Since image and videos are used to induce subjects' emotion, it was chosen a quiet room as the experimental environment to ensure that the effect of the screened videos is not compromised. The facial emotions recording system includes a color camera system (Kinect), thermal camera, eye tracker, illumination system, thermometer and humidity sensor.

Although thermal emissivity from the facial surface is relatively stable under illumination variations, it is sensitive to the temperature of the environment. Therefore, the temperature of the room is controlled during the experiments. 
The room temperature is between $20^{\circ} \mathrm{C}$ and $23^{\circ} \mathrm{C}$, and the humidity ranges between $30 \%$ and $40 \%$.

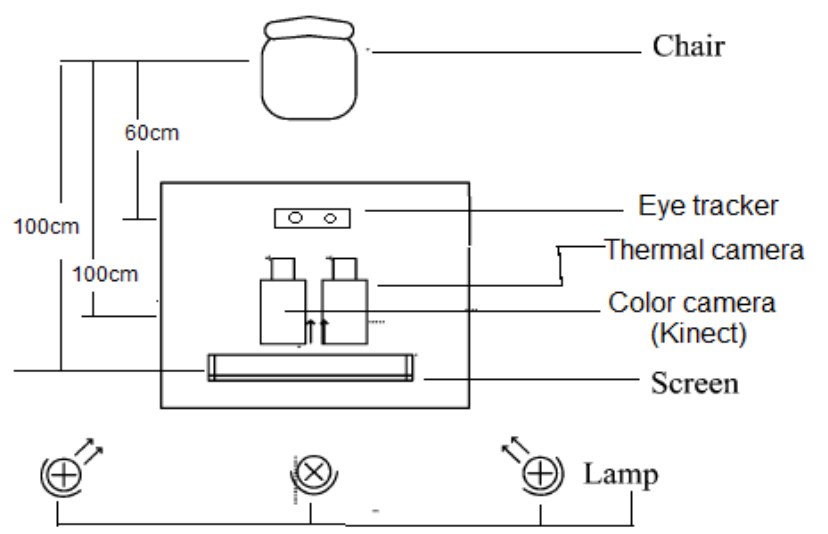

Fig. 4. Environment for experimental test.

\section{EXPERIMENTAL PROTOCOL}

The first experimental procedure was designed to evaluate social visual attention using eye tracker and Kinect integration; the second procedure was proposed to evaluate emotional variation (Arousal and Valence) using Kinect and thermal camera integration and, finally, the third procedure was designed to evaluate multisensorial emotion recognition using eye tracker, Kinect and thermal camera integration. This work had the approval of the Ethics Committee of UFES, number 1.121.638.

Procedure 1 - This procedure has the participation of eleven healthy adult volunteers (eight men and three women), with mean age of 28.27 years old $( \pm 5.33)$. Each volunteer is invited to sit comfortably in a chair positioned in front of a computer screen (Fig 4). In the first exhibition, the participant sees six pictures relative to human facial expressions (surprise, sadness, anger, disgust, fear and happiness), individually. Then, the participant should answer the emotion correspondent to the viewed emotional expression. With this procedure, are evaluated: a) which face regions the participant focused on to recognize an emotion and if he/she identifies the emotion correctly; b) which emotional facial expressions more attracts his/her attention; c) if the participant has difficulty to recognize the emotion required. For this, the number of times and the time of viewing the picture are assesed.

Procedure 2 - This procedure has the participation of sixteen healthy adult volunteers (twelve men and four women), with mean age of 28 years old $( \pm 5.32)$. The participant sees a set of six images (1024 x 768 pixels), being two of positive valence, two of negative valence and two neutral. The valence classification is based on a 1-9 scale, where scores were divided in three groups, with values greater than 7, lower than 5, and between 5 and 6.5 for pleasant, unpleasant and neutral pictures, respectively. The pictures are selected from an international database (IAPS - International Affective Picture System), commonly used in studies about emotions and attention (Lang 2008). The picture set is displayed five times and the time of exhibition of each image is ten seconds. The Therm-App sensor records images of each emotional facial expression performed by the volunteer, and an algorithm identifies the set of features related to expressions of each emotion, based on thermal variation for the valence and arousal detection.

Procedure 3 - This procedure has the participation of three volunteers, with age ranged between 6 to 11 years old. This procedure consists of the visualization by the volunteer of six emotion-inducing videos to evoke certain emotions (surprise, sadness, disgust, fear and happiness). Each volunteer sits down comfortably in a chair in front of both a screen and a box with the camera system (eye tracker, Kinect, thermal camera). This procedure evaluates multisensorial emotion recognition (focal attention and point of interest of the volunteer, facial expression recognition, valence recognition and emotional state).

\section{METHODS}

Three methods of integration were implemented in this work, which are Decision-Level, Feature-Level and Hybrid-Level.

\subsection{Decision-Level: eye tracker and Kinect Integration}

The integration of eye tracker and Kinect allows carrying out studies about focus of attention, in order to evaluate which parts of the face people focus on when they come to recognize expressions, and what stimuli generate an emotional reaction in a person.

Integration at the decision level was used in our research, since in this integration the two systems do not share features to obtain results. The integration is made with the results of the focus of attention, obtained by the eye tracker, and with the results of expression recognition, obtained by the Kinect. Fig. 5 shows the integration, where the focus of attention is detected on a face of a person to recognize a facial expression.

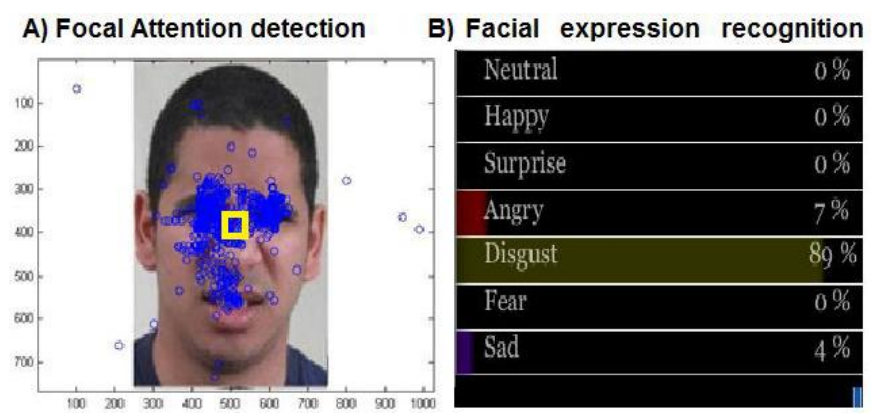

Fig. 5. Eye tracker- Kinect integration: A) Focus of attention detection; B) Facial expression recognition.

\subsection{Feature-Level: Kinect and thermal camera integration}

The integration of Kinect and thermal camera allows improving the thermal feature detection. The main problem to detect these thermal features of the face is the difficulty of segmenting the RoI, because in a thermal image it is difficult to detect regions such as eyes, mouth or nose. In contrast, in the color image, these facial features are easy to detect. The 
approach used in our research was to take the features obtained from the Kinect (AUs), and project them in the thermal image. Projecting the points of the AU in the thermal image is easier, in order to automatically segment the RoI and obtain the thermal features. A mathematical model for thisprojection was implemented using a camera calibration toolbox in MatLab. Any three-dimensional point $\left(X_{W}, Y_{W}, Z_{W}\right)$ in the scene can be taken to a camera coordinate system $\left(X_{C}\right.$, $Y_{C}, Z_{C}$ ), which is achieved with a rotation matrix $R$ and translation vector $T$, using (1).

$$
\left(\begin{array}{l}
X_{C} \\
Y_{C} \\
Z_{C}
\end{array}\right)=R\left(\begin{array}{c}
X_{W} \\
Y_{W} \\
Z_{W}
\end{array}\right)+T=\left(\begin{array}{lll}
r_{1} & r_{2} & r_{3} \\
r_{4} & r_{5} & r_{6} \\
r_{7} & r_{8} & r_{9}
\end{array}\right)\left(\begin{array}{c}
X_{W} \\
Y_{W} \\
Z_{W}
\end{array}\right)+\left(\begin{array}{c}
T_{x} \\
T_{y} \\
T_{z}
\end{array}\right)
$$

The values of $R$ and $T$ are known as extrinsic parameters, then this coordinated system in space must be taken into the two-dimensional space of the images, without considering the radial and tangential information. The coordinates of the image $\left(x_{b}, y_{b}\right)$ are calculated using (2).

$$
\left(\begin{array}{c}
x_{b} \\
y_{b} \\
1
\end{array}\right)=k\left(\begin{array}{ccc}
f_{c x} & s & C_{x} \\
0 & f_{c y} & C_{y} \\
0 & 0 & 1
\end{array}\right)\left(\begin{array}{c}
X_{c} \\
Y_{c} \\
Z_{c}
\end{array}\right)
$$

where: $f_{c x}$ and $f_{c y}$ are the focal distances expressed in pixels and include the focal length of the camera and the size in millimeters of the sensor $\left(S_{x}, S_{y}\right)$; and $C_{x}$ and $C_{y}$ are the optical center of the image. The value $\mathrm{s}$ is called framing, and most of the time it corresponds to an angle of $90^{\circ}$ and, therefore, its value is zero. The value $\mathrm{k}$ is a scaling factor, and the values $f_{c x}, f_{c y}, C_{x}, C_{y}, s$, and $k$ are known as intrinsic parameters. Fig. 6 shows the calibration process for AUs projection on the thermal image. The AUs points of the Kinect image are projected to its corresponding 3D model, then this model is transformed to the 2D model on the thermal image (Fig. 6). The integration of Kinect and thermal camera improves the thermal feature detection and the RoI segmentation.
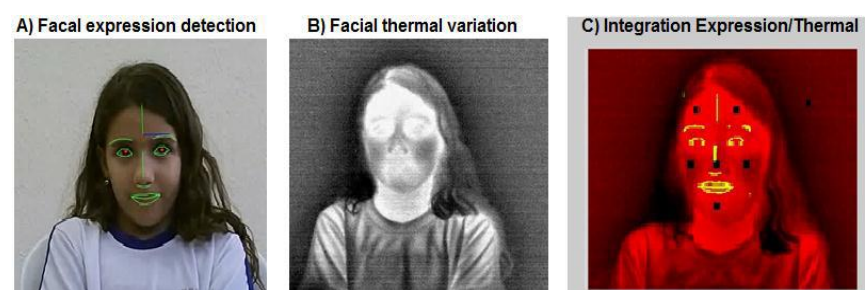

Fig. 6. Kinect and thermal camera integration: A) Facial expression detection; B) Facial thermal variation; C) Integration of AUs on thermal image.

\subsection{Hybrid-Level: eye tracker, Kinect and thermal camera Integration}

For the integration of the three systems, a hybrid-level integration was proposed to take advantage of the two integrations previously done (Kinect-eye tracker and thermal camera). The purpose of the integration of the three systems is to be able to give a more complete evaluation of the emotional state during the experimental stage by integrating the results of focus of attention, recognition of facial expressions and emotional variation of arousal and valence. The multisensorial integration allows realizing studies of social visual attention, recognition and expression of emotions, and to detect variation of the emotional state of a person. Fig. 7 shows the multisensorial integration implemented in this work, allowing focal attention detection, facial expression recognition, and estimation of emotional state.

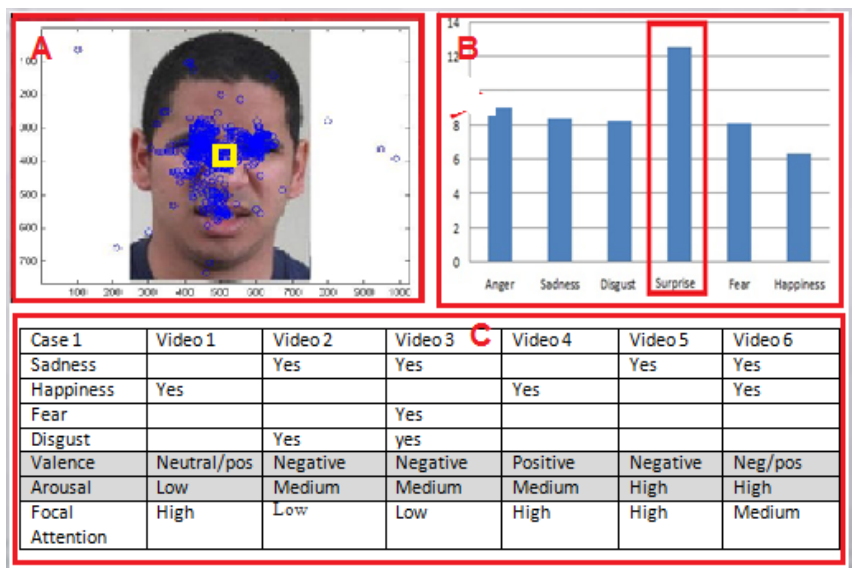

Fig. 7. Multisensorial integration: A) Focal attention detection, B) Facial expression recognition, C) Estimation of emotional state.

\section{RESULTS}

Table 1 shows the average time required by the participants to recognize the six facial expressions, the number of mistakes of the participants in the recognition of emotions from facial expressions, the percentage of expression recognition, and the percentage of focus detection for the experiment.

The average time required by the participants to recognize the six facial expressions was low for the happiness emotion $(6.3$ s) and high for the disgust (12.5 s). It was also noted that for the sixteen volunteers the number of mistakes for emotion recognition was higher for disgust (3 mistakes), while happiness had no mistake. Finally, the average of expression recognition was $70 \%$, and the average of focus of attention was $78 \%$.

Table 1. Facial expressions recognition.

\begin{tabular}{|l|l|l|l|l|}
\hline $\begin{array}{c}\text { Emotion } \\
\text { expressions }\end{array}$ & $\begin{array}{c}\text { Time to } \\
\text { recognize } \\
(\mathrm{s})\end{array}$ & $\begin{array}{c}\text { Number } \\
\text { of } \\
\text { mistakes }\end{array}$ & $\begin{array}{c}\text { Expression } \\
\text { recognition }\end{array}$ & $\begin{array}{c}\text { Focus of } \\
\text { attention }\end{array}$ \\
\hline Anger & $9.7 \pm 5.1$ & 1 & $58 \%$ & $83 \%$ \\
\hline Sadness & $8.7 \pm 2.5$ & 1 & $50 \%$ & $79 \%$ \\
\hline Surprise & $8.8 \pm 3.9$ & 2 & $85 \%$ & $72 \%$ \\
\hline Disgust & $12.5 \pm 7.2$ & 3 & $75 \%$ & $68 \%$ \\
\hline Fear & $8.9 \pm 3.5$ & 1 & $64 \%$ & $79 \%$ \\
\hline Happiness & $6.3 \pm 0.6$ & 0 & $89 \%$ & $87 \%$ \\
\hline
\end{tabular}


Table 2 shows the results from the experiments. For valence, the most important variation was observed in the nose; for positive valence, the temperature decreases up to $4 \%$, and for negative valence, it can increase up to $8 \%$ in relation to images of neutral valence. On the other hand, for arousal, an increase from $3 \%$ to $4 \%$ in cheek and forehead temperature was observed; the nose also had a slightly lower increase of $2 \%$.

Table 2. Percentage of RoIs thermal variation in arousal and valence.

\begin{tabular}{|l|l|l|l|l|l|l|}
\hline Emotion & Nose & Chin & $\begin{array}{c}\text { Right } \\
\text { cheek }\end{array}$ & $\begin{array}{c}\text { Left } \\
\text { cheek }\end{array}$ & $\begin{array}{c}\text { Right } \\
\text { forehead }\end{array}$ & $\begin{array}{c}\text { Left } \\
\text { forehead }\end{array}$ \\
\hline $\begin{array}{l}\text { Positive } \\
\text { Valence }\end{array}$ & $-4 \%$ & $2 \%$ & $2 \%$ & $2 \%$ & $3 \%$ & $3 \%$ \\
\hline $\begin{array}{l}\text { Neutral } \\
\text { Valence }\end{array}$ & $1 \%$ & $0 \%$ & $0 \%$ & $1 \%$ & $1 \%$ & $0 \%$ \\
\hline $\begin{array}{l}\text { Negative } \\
\text { Valence }\end{array}$ & $+8 \%$ & $2 \%$ & $2 \%$ & $2 \%$ & $3 \%$ & $3 \%$ \\
\hline $\begin{array}{l}\text { Low } \\
\text { Arousal }\end{array}$ & $1 \%$ & $0 \%$ & $-1 \%$ & $-1 \%$ & $0 \%$ & $0 \%$ \\
\hline $\begin{array}{l}\text { High } \\
\text { arousal }\end{array}$ & $+2 \%$ & $1 \%$ & $+3 \%$ & $+3 \%$ & $+4 \%$ & $+4 \%$ \\
\hline
\end{tabular}

Table 3 shows the emotions that each video is intended to evoke. In this case, three participants were selected and evaluated as a study case.

Table 3. Emotions that each video is intended to evoke.

\begin{tabular}{|l|l|l|l|l|l|l|}
\hline $\begin{array}{c}\text { Emotion } \\
\text { intended } \\
\text { to evoke }\end{array}$ & $\begin{array}{c}\text { Video } \\
1\end{array}$ & $\begin{array}{c}\text { Video } \\
2\end{array}$ & $\begin{array}{c}\text { Video } \\
3\end{array}$ & $\begin{array}{c}\text { Video } \\
4\end{array}$ & $\begin{array}{c}\text { Video } \\
5\end{array}$ & $\begin{array}{c}\text { Video } \\
6\end{array}$ \\
\hline Sadness & - & Yes & Yes & - & Yes & Yes \\
\hline Happiness & Yes & - & - & Yes & - & Yes \\
\hline Fear & - & Yes & Yes & - & - & - \\
\hline Disgust & - & Yes & yes & - & - & - \\
\hline Valence & pos & Neg & Neg & Pos & Neg & $\begin{array}{l}\text { Neg/ } \\
\text { pos }\end{array}$ \\
\hline Arousal & Low & High & High & High & High & Med \\
\hline $\begin{array}{l}\text { Focal } \\
\text { Attention }\end{array}$ & High & Low & Low & High & Med & Med \\
\hline
\end{tabular}

Case 1. Table 4 indicates the emotions recognized by the multisensorial system for volunteer 1 . It was observed that Video 1 evokes happiness, neutral to positive valence and high focal attention. Video 2 evoked sadness and disgust, in which the valence was negative, the arousal was medium and there was a low focus of attention. Video 3 evoked mostly sadness, fear and disgust, in which valence was negative, arousal was medium, and the focus of attention was low. Video 4 showed a high level of happiness, positive valence, a medium arousal and high attention. In Video 5, the main emotion was sadness, there was negative valence, the level of arousal was high and the focus of attention was high. Finally, the Video 6 in the first part of video sadness, and the last part happiness, with negative and positive valence respectively, a high arousal and a medium focus of attention.
Table 4. Recognition of emotions evoked for each video by Volunteer 1.

\begin{tabular}{|l|l|l|l|l|l|l|}
\hline \multicolumn{1}{|c|}{ Case 1 } & $\begin{array}{l}\text { Video } \\
1\end{array}$ & $\begin{array}{c}\text { Video } \\
2\end{array}$ & $\begin{array}{c}\text { Video } \\
3\end{array}$ & $\begin{array}{c}\text { Video } \\
4\end{array}$ & $\begin{array}{c}\text { Video } \\
5\end{array}$ & $\begin{array}{c}\text { Video } \\
6\end{array}$ \\
\hline Sadness & - & Yes & Yes & - & Yes & Yes \\
\hline Happiness & Yes & - & - & Yes & - & Yes \\
\hline Fear & - & - & Yes & - & - & - \\
\hline Disgust & - & Yes & Yes & - & - & - \\
\hline Valence & $\begin{array}{l}\text { Neut/ } \\
\text { pos }\end{array}$ & Neg & Neg & Pos & Neg & $\begin{array}{l}\text { Neg/ } \\
\text { pos }\end{array}$ \\
\hline Arousal & Low & Med & Med & Med & High & High \\
\hline $\begin{array}{l}\text { Focal } \\
\text { Attention }\end{array}$ & High & Low & Low & High & High & Med \\
\hline
\end{tabular}

Case 2. Table 5 indicates the emotions recognized by the multisensorial system for volunteer 2 . It was observed that video 1 evokes happiness, neutral valence, low arousal and high focus of attention. Video 2 evoked happiness and disgust, the valence was neutral, there was low arousal and medium focus of attention. Video 3 evoked happiness and fear, while valence was neutral; there was low arousal and low focus of attention. Video 4 showed a high level of happiness, positive valence, a low arousal and high focus of attention. In Video 5, the main emotion was sadness, negative valence, the level of arousal was medium and the focus of attention was high. Finally, the Video 6 showed happiness, with negative and positive valence, medium arousal and high focus of attention.

Table 5. Recognition of emotions evoked for each video by Volunteer 2.

\begin{tabular}{|l|l|l|l|l|l|l|}
\hline \multicolumn{1}{|c|}{ Case 2} & $\begin{array}{c}\text { Video } \\
1\end{array}$ & $\begin{array}{c}\text { Video } \\
2\end{array}$ & $\begin{array}{c}\text { Video } \\
3\end{array}$ & $\begin{array}{c}\text { Video } \\
4\end{array}$ & $\begin{array}{c}\text { Video } \\
5\end{array}$ & $\begin{array}{c}\text { Video } \\
6\end{array}$ \\
\hline Sadness & - & - & - & - & Yes & - \\
\hline Happiness & Yes & Yes & Yes & Yes & - & Yes \\
\hline Fear & - & - & Yes & - & - & - \\
\hline Disgust & - & Yes & - & - & - & - \\
\hline Valence & Neut & Neu & Neu & Pos & Neg & $\begin{array}{l}\text { Neg/ } \\
\text { pos }\end{array}$ \\
\hline Arousal & Low & Low & Low & Low & Med & Med \\
\hline $\begin{array}{l}\text { Focal } \\
\text { Attention }\end{array}$ & High & Med & Low & High & High & High \\
\hline
\end{tabular}

Case 3. Table 6 indicates the emotions recognized by the multisensorial system for volunteer 3 . It was observed that video 1 evokes happiness, positive valence, low arousal and high focus of attention. Video 2 evoked disgust, the valence was neutral, with low arousal and high focus of attention. Video 3 evoked fear, while valence was neutral, with low arousal and medium focus of attention. Video 4 showed happiness, positive valence, medium arousal and high focus of attention. In Video 5, the main evoked emotions were sadness and disgust, with negative valence, high level of arousal and medium focus of attention. Finally, Video 6 showed sadness, with negative valence, high arousal and low focus of attention. 
Table 6. Recognition of emotions evoked for each video by Volunteer 3.

\begin{tabular}{|l|l|l|l|l|l|l|}
\hline \multicolumn{1}{|c|}{ Case 3} & $\begin{array}{l}\text { Video } \\
1\end{array}$ & $\begin{array}{c}\text { Video } \\
2\end{array}$ & $\begin{array}{c}\text { Video } \\
3\end{array}$ & $\begin{array}{c}\text { Video } \\
4\end{array}$ & $\begin{array}{c}\text { Video } \\
5\end{array}$ & $\begin{array}{c}\text { Video } \\
6\end{array}$ \\
\hline Sadness & - & - & - & - & Yes & Yes \\
\hline Happiness & Yes & - & - & Yes & - & - \\
\hline Fear & - & - & Yes & - & - & - \\
\hline Disgust & - & Yes & - & - & Yes & - \\
\hline Valence & Pos & Neut & Neut & Pos & Neg & Neg \\
\hline Arousal & Low & Low & Low & Med & High & High \\
\hline $\begin{array}{l}\text { Focal } \\
\text { Attention }\end{array}$ & High & High & Med & High & Med & Low \\
\hline
\end{tabular}

\section{DISCUSSION AND CONCLUSIONS}

A multisensorial integration strategy was presented in this work. The multisensorial approach presents challenges related to the fusion of single signals from different sensors, dimensionality of the feature space, and incompatibility of collected signals in terms of time resolution and format (Pantic 2003), (Al Osman, 2016), (Lingenfelser, 2011) and (Wagner, 2011).

The strategies presented in this work allowed integrating such heterogeneous devices like eye tracker, Kinect and thermal camera into an all-in-one system. The main difficulties in the integration were the range of operation of each equipment and the difficulty of synchronizing the data captured by different computers. These problems were solved, firstly by changing the setup for the experimental tests, and secondly with a manual synchronization of the videos. The integration of eye tracker and Kinect allowed performing joint studies of focus of attention in recognition of facial expressions and valence, while the Kinect-thermal camera integration allowed using the $\mathrm{AU}$ in the thermal images, which improved the detection and segmentation of FT-RoI in the thermal image.

The major difficulties of the experiments were that the data acquisition for the three sensors is not synchronized and, thus, a manual synchronization process was required. On the other hand, the features of the thermal camera (vision mode, image resolution and sensitivity) were not suitable for the proposed procedures. also, the eyes' regions are small and very limited to be analyzed. Despite the difficulties presented, the system has potential to be used in applications of emotion recognition, although more investigations are necessary.

Finally, with the system developed here, emotions of people can be analyzed by facial features using contactless sensors in semi-structured environments, such as clinics, laboratories, or classrooms. This system also presents the potential to become an embedded tool in robots to endow these machines with an emotional intelligence for a more natural interaction with humans.

\section{ACKNOWLEDGEMENTS}

The authors would like to thank the Federal University of Espirito Santo (UFES), CNPq and CAPES (Brazil) for financial support and scholarships. This study was financed in part by CAPES - Finance Code 88887.095636/2015-01.

\section{REFERENCES}

Alexandre, L. A.; Campilho, A. C.; Kamel, M. (2001). On combining classifiers using sum and product rules. Pattern Recognition Letters, vol. 22, pp. 1283-1289.

Al Osman, H.; Dong, H.; El Saddik, A. (2016). Ubiquitous biofeedback serious game for stress management. IEEE Access, vol. 4, pp. 1274-1286.

Bartlett, M.; Hager, J.; Ekman P.; Sejnowski, T. (1999) Measuring facial expressions by computer image analysis. Psychophysiology, 36:253-264.

Bengoechea, J.; Villanueva, A., Cabeza, R. (2012). Hybrid eye detection algorithm for outdoor environments. Proceedings of the 2012 ACM conference on ubiquitous computing. ACM, New York, pp 685-688.

Duchowski, A. T.; Vertegaal, R. (2000) Eye-based interaction in graphical systems: theory and practice. Course 05, SIGGRAPH 2000. ACM, New York.

Ekman, P. Friesen, W. (1978). The Facial Action Coding System: a technique for the measurement of facial movement. Consulting psychologists Press, Inc., San Francisco, CA.

Essa, I.; Pentland, A. (1997). Coding, analysis, interpretation, and recognition of facial expressions. IEEE Trans. on Pattern Analysis and Machine Intell., 19(7):757-763.

Khan, M.; Ward, R.; Ingleby, M. (2009). Classifying pretended and evoked facial expressions of positive and negative affective states using infrared measurement of skin temperature, Trans. Appl. Percept., vol.6. 1-22.

Kim, J.; André, E.; Rehm, M.; Vogt, T.; Wagner, J. (2005). Integrating information from speech and physiological signals to achieve emotional sensitivity. Proc. INTERSPEECH, Lisboa, Portugal.

Lingenfelser, F.; Wagner, J.; André, E. A (2011). systematic discussion of fusion techniques for multi-modal affect recognition tasks. Proceedings of the 13th international conference on multimodal interfaces.

Mase, K.; (1991). Recognition of facial expression from optical flow. IEICE Transactions, E. 74(10):3474-3483.

Pantic, M.; Rothkrantz, L. J. (2003). Toward an affect-sensitive multimodal human-computer interaction. Proceedings of the IEEE, vol. 91, pp. 1370-1390.

Pavlidis, I.; Eberhardt, N. L.; Lev-Ine, J. A. (2002). Human behaviour: Seeing through the face of deception. Nature, 4, 35. http://dx.doi.org/10.1038/415035a.

Rivera, H. (2017). Development of a multisensorial system for emotion recognition, Master, Federal University of Espírito Santo, Brazil.

Sugimoto, Y.; Yoshitomi, Y.; To-Mita, S. (2000). A method of detecting transitions of emotional states using a thermal facial im-age based on a synthesis of facial expres-sions. Robotics Autonom. Syst. 31, 147-160.

Veltman, J. A.; Vos, W. K. (2005). Facial temperature as a measure of operator State. In Paper presented at the 11th international conference on human- computer interaction, 22-27 July, Las Vegas-Nevada, USA.

Wagner, J.; Andre, E.; Lingenfelser, F.; Kim, J. (2011). Exploring fusion methods for multimodal emotion recognition with missing data. IEEE Transactions on Affective Computing, vol. 2, pp. 206-218. 